\title{
Defining Area Mass Index (AMI) Using 3D Body Scanning as an Improvement of BMI
}

\author{
Elmar H. SCHLICH ${ }^{1 a}$, Michaela SCHLICH \\ a Justus Liebig University Giessen, Germany \\ bUniversity Koblenz-Landau, Koblenz, Germany
}

\begin{abstract}
The surface area of the human body determines the amount of heat exchanged with the environment, which is a major element of the energy balance equation as it relates to weight management. The traditional Body Mass Index $(B M I)$ calculation uses the body height squared as its denominator (unit: $\mathrm{m}^{2}$ ). This approximation does not consider that the heat exchange between the environment and a person with a trim build is different than that of a person with a stout build, because the specific surface size of a human body depends on its shape.

Using a laser body scanning machine, 188 people were measured to obtain reliable data of their heat exchanging surface in order to calculate and define the new measurement of Area Mass Index (AMI). As expected, the results of the $A M I$ calculations deviated significantly from the calculations of the $B M I$. Actually, the detected deviation was not a constant but dependent upon the $B M I$ itself. The value of the $A M I$ decreased as the $B M I$ value increased. This paper presents the basics of laser body scanning and provides the results of these series of tests.

The $A M I$ and the Specific Body Surface (SBS) are important factors in calculating the amount of heat a body exchanges with the environment. Using a standard regression analysis, the linear function of $A M I$ $=\mathrm{f}(B M I)$ is calculated separately for men and women. As expected, the hyperbolic trend of Specific Body Surface (SBS) and BMI is clearly demonstrated. These findings prove that people with higher $B M / s$ always face disadvantages in reducing body mass because of having a lower SBS. On the other hand, slim people have principal problems gaining weight because of their higher SBS. The traditional role of $B M I$ in weight management in consultation with nutritionists could be improved by means of an $A M I$ conversion factor, which can be derived by using highly sophisticated 3D body scanning.
\end{abstract}

Keywords: 3D body scan, Area Mass Index, $A M I$, body mass index, $B M I$, human energy balance.

Table 1: Abbreviations and units

\begin{tabular}{|l|l|l|}
\hline Abbreviation & Explanation & Unit \\
\hline$A$ & area, body surface & $\mathrm{m}^{2}$ \\
\hline$A M I$ & Area Mass Index & $\mathrm{kg} / \mathrm{m}^{2}$ \\
\hline$B M I$ & Body Mass Index & $\mathrm{kg} / \mathrm{m}^{2}$ \\
\hline$D$ & diameter & $\mathrm{m}$ \\
\hline$d$ & differential & - \\
\hline $\mathrm{dQ} / \mathrm{d} t$ & heat flux & $\mathrm{W}, \mathrm{kW}$ \\
\hline$E$ & energy & $\mathrm{J}, \mathrm{kJ}$ \\
\hline$E_{S}$ & energy of system & $\mathrm{J}, \mathrm{kJ}$ \\
\hline$H$ & body height & $\mathrm{m}$ \\
\hline$m$ & body mass & $\mathrm{kg}$ \\
\hline$n$ & number of test subjects & - \\
\hline$P$ & power, energy flux & $\mathrm{W}, \mathrm{kW}$ \\
\hline$Q$ & heat & $\mathrm{J}, \mathrm{kJ}$ \\
\hline $\mathrm{R}^{2}$ & coefficient of determination & - \\
\hline$S B S$ & Specific Body Surface & $\mathrm{m}^{2} / \mathrm{kg}$ \\
\hline$T$ & temperature & $\mathrm{K},{ }^{\circ} \mathrm{C}$ \\
\hline$t$ & time & $\mathrm{s}$ \\
\hline$V$ & volume & $\mathrm{m}^{3}$ \\
\hline$\alpha$ & heat transfer coefficient & $\mathrm{W} / \mathrm{m}^{2} / \mathrm{K}$ \\
\hline$\Delta$ & difference & - \\
\hline$\Delta T$ & difference of temperatures & $\mathrm{K}$ \\
\hline
\end{tabular}

\footnotetext{
1 elmar.schlich@uni-giessen.de; +49-641-9939350; http://www.uni-giessen.de/fbr09/pt
} 


\section{Introduction and theoretical background}

Human energy turnover plays a large role in weight management. This process is in accordance with the basic principles of energy turnover, which include the 1st and 2nd laws of thermodynamics [1]. If a human body is considered an open thermodynamic system, then the energy balance is calculated in accordance with equation 1 :

$$
\mathrm{d} E_{\mathrm{S}}=+E_{\text {Input }}-E_{\text {Output }}
$$

Obviously, there are three possible cases identified for adults (tab. 2):

Table 2: Principle cases of the human energy balance equation for adults

\begin{tabular}{|l|l|}
\hline In/Out proportion & Result in relation to body mass \\
\hline$E_{\text {Input }}=E_{\text {Output }}$ & $\begin{array}{l}\text { The energy of the human system does not change, } \\
\text { and body mass remains constant. }\end{array}$ \\
\hline$E_{\text {Input }}>E_{\text {Output }}$ & $\begin{array}{l}\text { The energy of the human system increases, and } \\
\text { the body gains weight as a store of surplus energy. }\end{array}$ \\
\hline$E_{\text {Input }}<E_{\text {Output }}$ & $\begin{array}{l}\text { The energy of the human system decreases, and } \\
\text { the body loses weight thus compensating for lack } \\
\text { of energy supply. }\end{array}$ \\
\hline
\end{tabular}

Energy input is controlled by the amount and types of daily food intake. To successfully manage body weight all diet recommendations focus on the quantity and quality of food intake as part of the human energy balance equation. Energy output is also an essential part of the human energy balance and is primarily dictated by maintaining body temperature at the necessary level of $\sim 36.6^{\circ} \mathrm{C}$, while facing an external temperature that is much lower in most cases. Hence, the basal metabolic rate ensures a permanent heat transfer from the body to the environment to stabilize the thermal imbalance. This stabilization is required for the survival of human life. The transient heat flux, $\mathrm{d} Q / \mathrm{d} t$, from the body to its surroundings, is dependent on the heat transmission coefficient, $\alpha$, the surface area of the human body, $A$, and the difference of temperatures, $\Delta T$ [2]. This fundamental relationship of heat transfer is shown by equation 2:

$$
\mathrm{dQ} / \mathrm{d} t=\alpha \cdot A \cdot \Delta T
$$

The following example illustrates the significance of this equation. If we assume a daily energy input of a subject of $E=10 \mathrm{MJ}$, then this corresponds with a power generation of $P=115.7 \mathrm{~W}^{2}$ on average. In the case of a well-balanced energy turnover of $E_{\text {Input }}=E_{\text {Output }}$, this power has to leave the human body. According to the $2^{\text {nd }}$ law of thermodynamics, the main energy output consists of a steady heat transfer to the external surroundings. Other smaller components of energy transfer to the external environment include the thermal energy of the breath, excretion, and enthalpy of perspiration. All standard activities of the muscles, such as heartbeats, breathing, or digestion, are converted by dissipation into thermal energy. If the basal metabolic rate is $60 \%$ of this total power generation, then a steady daily heat flux as a mean value of $\mathrm{dQ} / \mathrm{d} t=70 \mathrm{~W}$ must be transferred to the surrounding environment. Given the heat transmission coefficient, $\alpha$, and the difference between temperatures, $\Delta T$, which is not dependent on the shape of the human body, the body surface, $A$, turns out to be the essential factor of energy output in terms of heat transfer.

It is obvious and well known, because of work in the field of anthropometry [3] as well as by the historical definition of body mass index BMI [4] and its longtime scientific elaboration, that the surface of the body as a geometrical parameter determines the amount of external heat transfer whereas the body mass as volumetric parameter, especially the muscle, is the heat generating source. The relationship between heat transfer and heat source can be described using the following equation: true body surface, $A$, divided by body mass, $m$. We define this relationship as the Specific Body Surface (SBS) using the unit of measurement $\mathrm{m}^{2} / \mathrm{kg}$, which can be understood as being the reciprocal of $B M I$. In this case, BMI would indeed be based on the true surface of the human body. The SBS represents a hyperbolic function of the body mass as shown by equation 3:

$$
S B S=A / m
$$

\footnotetext{
${ }^{2}$ Calculation pursuant to: $P=E / t=10,000 \mathrm{~kJ} / 24 \mathrm{~h} / 3,600 \mathrm{~s}=0.1157 \mathrm{~kW}=115.7 \mathrm{~W}$.
} 
In contrast, the traditional $B M I$ calculation, shown in equation 4 , takes the square of body height $\left(H^{2}\right)$ as the denominator which could be understood as approximate value for the body surface $A$. This equation originally was developed in the year 1871 [4], and it is still in use [3]:

$$
B M I=m / H^{2}
$$

However, this simple approximation does not consider that in terms of the heat exchange equation, trim bodies react differently than do the bodies of people with a stout build. The surface, $A$, depends on the shape of a body even if the body mass and/or body volume are identical to other bodies. People with trim figures are expected to have a higher SBS when compared with more stout people. Table 3 demonstrates this fundamental concept with a simple example using trim and stout cylinders to calculate the $B M I$ and SBS. In this case, both cylinders have a mass of $80 \mathrm{~kg}$ and a volume of $80 \mathrm{I}$.

Table 3: The BMI and SBS of trim and stout cylinders, both with $m=80 \mathrm{~kg}$ and $V=80 \mathrm{I}$

\begin{tabular}{|l|l|l|l|l|l|}
\hline Shape & $D[\mathrm{~m}]$ & $H[\mathrm{~m}]$ & $B M I\left[\mathrm{~kg} / \mathrm{m}^{2}\right]$ & $A\left[\mathrm{~m}^{2}\right]$ & $S B S\left[\mathrm{~m}^{2} / \mathrm{kg}\right]$ \\
\hline Trim cylinder & 0.226 & 2 & 20 & 1.5 & 0.01875 \\
\hline Stout cylinder & 0.26 & 1.5 & 35.56 & 1.33 & 0.01662 \\
\hline
\end{tabular}

This calculation proves the declining SBS with an increasing BMI using admittedly simple cylinder dimensions as an example. Similarly, the decline of $S B S$ with an increasing $B M I$ in human beings is expected, most likely with some variation between adult men and women.

With regard to human bodies, it would be exciting to have an exact measurement of the true surface area of test subjects in order to calculate the human SBS as the Specific Body Surface accordant with equation 3. The reciprocal of this measured human SBS establishes a distinctive value that we entitle Area Mass Index ( $\boldsymbol{A} M \boldsymbol{M})$ using the unit $\mathrm{kg} / \mathrm{m}^{2}$, which is the same unit used in traditional $B M I$ (equation $5)$.

$$
A M I=S B S^{-1}=m / A
$$

Using the $A M I$ data, it may be possible to determine out whether there is indeed a discrepancy between the traditional $B M I$ and $A M I$ and whether this differential declines with increasing $B M I$ as expected by the theory put forth. Additionally, a conversion function, such as $A M I=f(B M I)$, should be developed, separately for men and women, to estimate the true surface area of human beings as best as possible. Such a function would establish a better understanding of the fundamental difference in body mass management between trim people and people of stout build and would consider that their energy balances obey different rules based on the laws of thermodynamics.

\section{Materials and methods}

The true body surface measurement performed in this research is based on the technology of the 3D-Body-Scan [5] mobile system. This system is currently being used by the Hohensteiner Institute [6] and Human Solutions $\mathrm{GmbH}$ [7] in Germany to obtain a large amount of statistical data on the body dimensions of the German population [8]. This large-scale body dimension survey is actually being performed to create accurate clothing measurements for the apparel industry. For our current research, to measure the true body surface, $A$, we were able to use the mathematical analysis of the 3D-Body-Scan data. The test location for this research was the process engineering and thermodynamics laboratory at Justus Liebig University Giessen.

The 3D-Body-Scan is a rapid and contact-free process that scans the entire body using red laser beams. The scanner system consists of four vertical columns that are arranged in a square with the test subject in the center. Each column is equipped with a laser emission system and two cameras carried in a runner. The optical system is calibrated in both vertical and horizontal directions to set the four laser beams in accurate coplanarity. The quality of calibration is tested by dummies with well-known dimensions.

At the beginning of the test procedure, all lasers are positioned on the top of the column. The test subjects are wearing nonreflective undergarments and bathing caps and are positioned precisely in the center. The four laser systems simultaneously scan down to the floor and then up again. A single scanning operation takes only 12 seconds.

Four different positions of each test subject are analyzed including standing relaxed with unbent hands (pos. 1), standing at attention with clenched fists (pos. 2), standing with the left arm horizontally stretched and the right elbow angled (pos. 3), and sitting with both elbows angled (pos. 4) (see Figure 1). These four positions guarantee a complete scan of the entire human body and its dimensions. The process of scanning all four scanning positions takes approximately 2 minutes in total. 


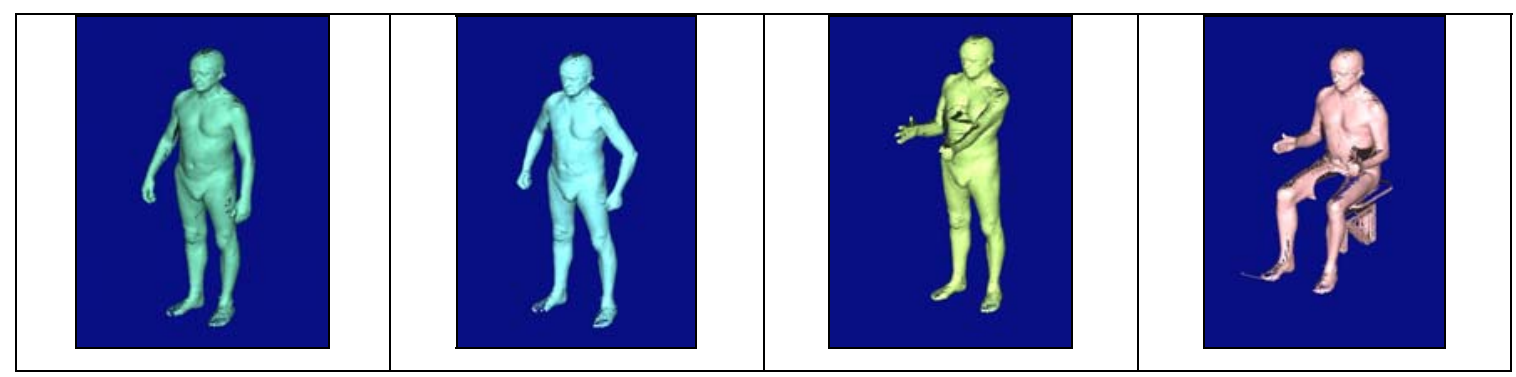

Fig. 1: Posture of the four test positions for 3D-Body-Scan

For a single test subject, the entire procedure takes approximately 10-15 minutes in total. This time includes the time to change clothing, to measure the body weight, and finally to record age, ethnic origin, and gender. For the final result, a three-dimensional cluster of points is generated and assigned to one real subject. Each avatar (Figure 2) can be plotted on the screen as a polygon and then analyzed by special software to receive all the metric dimensions and the surface area, $A[3]$.

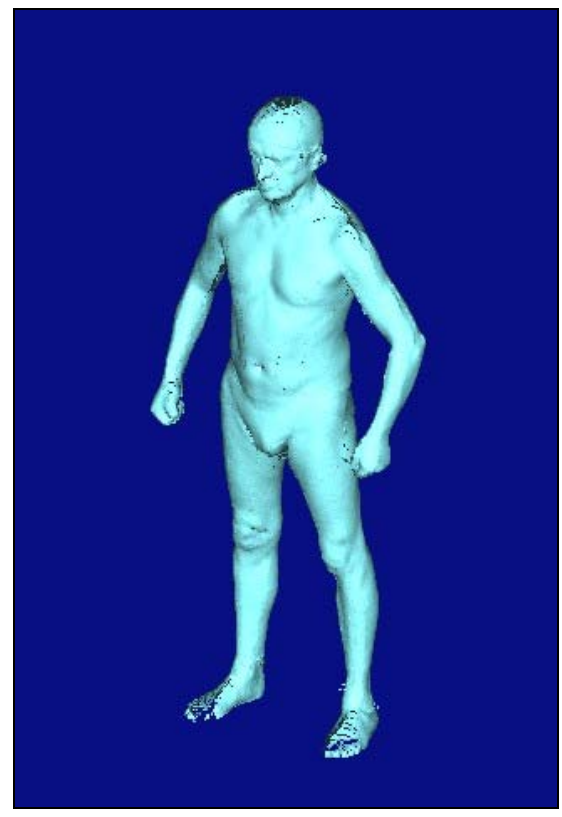

Fig. 2: The avatar as the final result of the 3D-Body-Scan

Finally, anonymous data were made available for the current study and transferred to a MS Excel file. The data included serial number, gender, age, ethnic origin, body height, $H$, body mass, $m$, and true body surface area, $A$. With this data, the BMIs, AMIs, and SBSs of each male and female test subject, were calculated using equations 3,4 and 5 , respectively. Then, the functions of $A M I=f(B M I)$ and its reciprocal, $S B S=f(B M I)$, were determined using a MS Excel spreadsheet. Finally, an ordinary regression analysis by means of linear interpolation was performed, yielding a linear equation and a coefficient of determination, $\mathrm{R}^{2}$.

\section{Results and discussion}

Altogether, the anonymous data of 188 subjects were made available as a result of the measurement campaign at Justus Liebig University Giessen performed in December 2007. Seven of these records were the measurements of male and female children between five and twelve years old. One hundred thirty-two test subjects were female between 20 and 84 years old, and 49 test subjects were male between 21 and 68 years old. The data of the seven children were not used.

\section{Female test subjects}

The function of $A M I=\mathrm{f}(B M I)$ using the data from the female test subjects is presented in Figure 3 (see next page). Additionally, the theoretical correlation of $A M I$ as it relates to $B M I$ is drawn as a dotted line. Figure 3 indicates a strong deviation between $A M I$ and $B M I$, as was expected. More interesting is the decline of this deviation as $B M I$ increases, as can be seen in the diagram. The linear regression analysis of these data gives a clear correlative function as shown in equation 6 : 


$$
A M I=0.865 \cdot B M I+18.56
$$

The coefficient of determination of that linearization is extremely high with $R^{2}=0.942$. Figure 4 (see next page) shows the correlation of the Specific Body Surface (SBS) with traditional BMI. As hypothesized, we find a declining hyperbolic trend when looking at the SBS as a function of BMI. The associated nonlinear interpolation yields equation 7 :

$$
S B S=0.141 \cdot B M r^{0.54}
$$

The coefficient of determination of that interpolation is similarly high, with $R^{2}=0.947$. The data of female test subjects show a maximum value of $S B S=0.031 \mathrm{~m}^{2} / \mathrm{kg}$ with a very low $B M I$ level and values of less than $0.02 \mathrm{~m}^{2} / \mathrm{kg}$ of adipose BMI. It seems quite remarkable that the specific body surface of trim female figures is up to $50 \%$ higher when compared with women with stout figures.

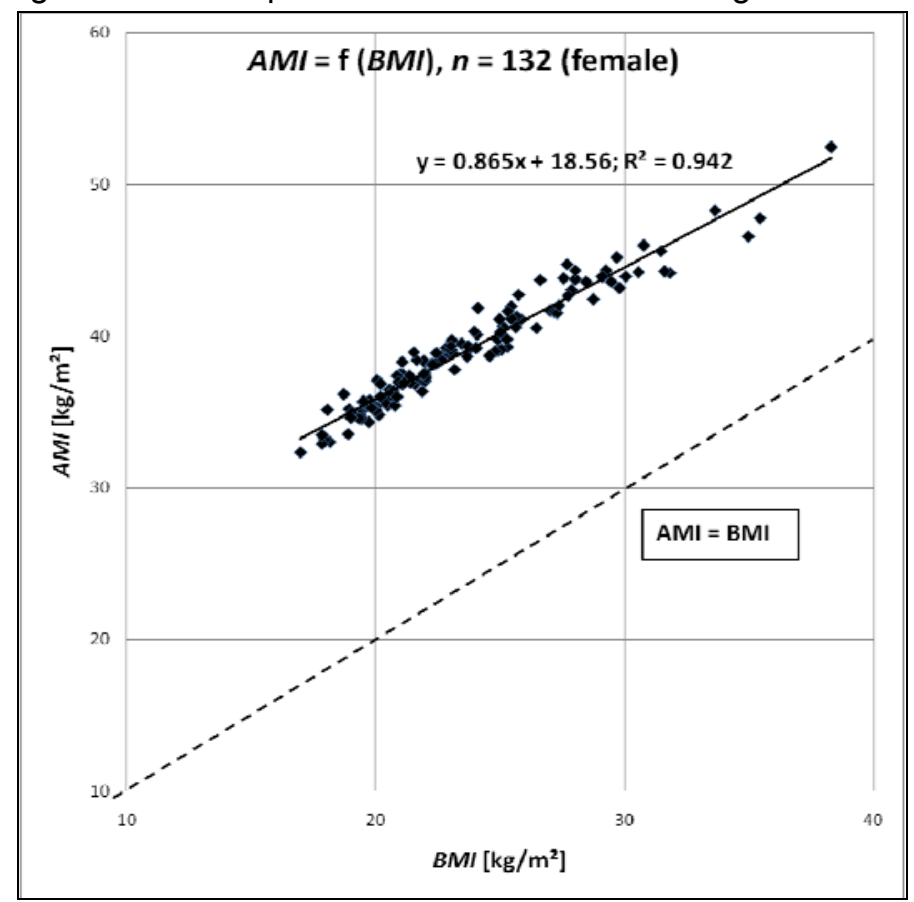

Fig. 3: Area Mass Index (AMI) as a function of traditional BMI, $n=132$ (female)

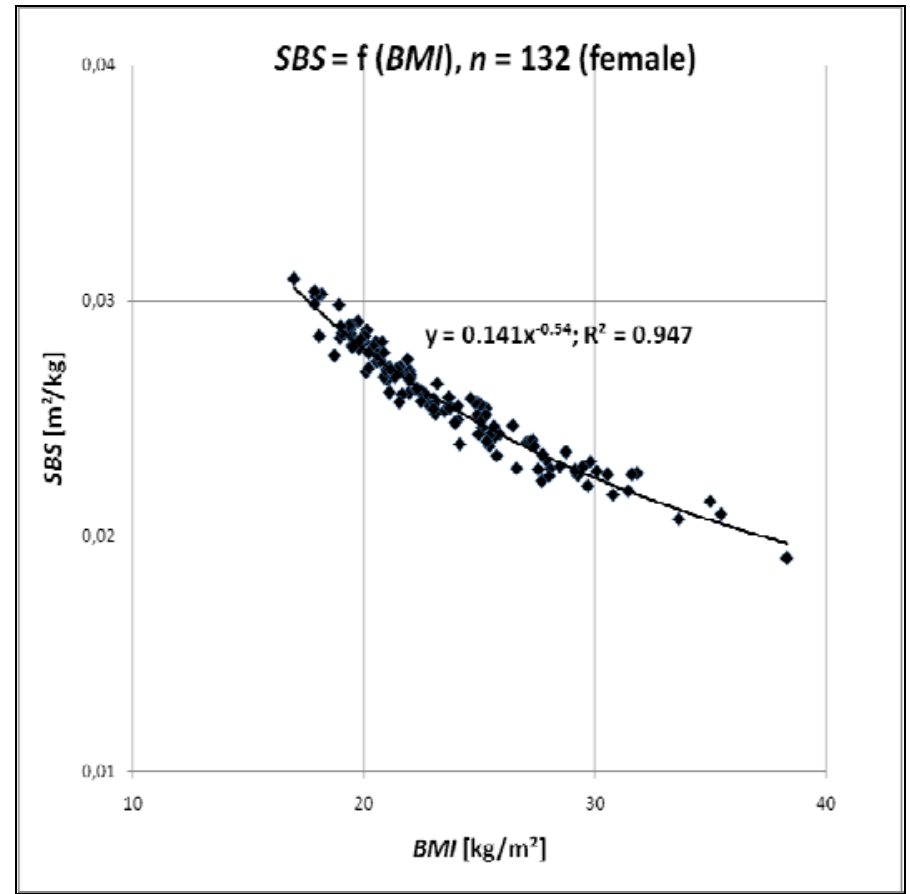

Fig. 4: Specific Body Surface (SBS) as a function of traditional BMI, $n=132$ (female) 


\section{Male test subjects}

The function of $A M I=\mathrm{f}(B M I)$ as result of the male test subject data is presented in Figure 5.

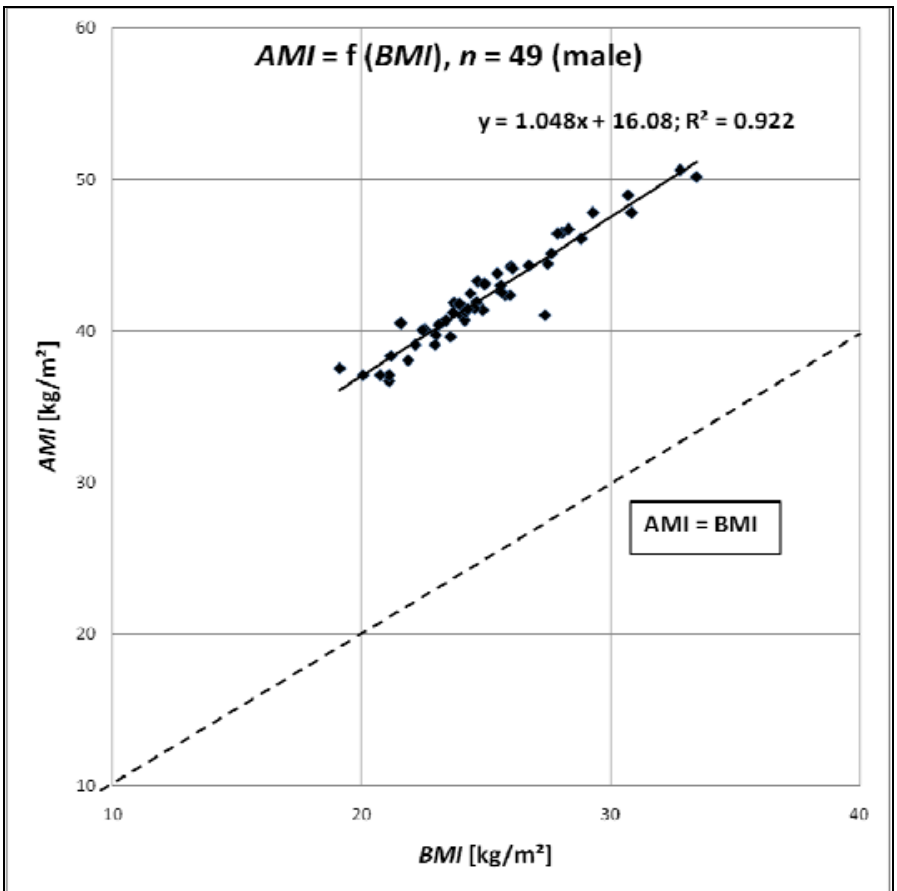

Fig. 5: Area Mass Index (AMI) as a function of traditional BMI, $n=49$ (male)

Again, the theoretical correlation between $A M I$ and $B M I$ is drawn as a dotted line. Figure 5 also indicates a strong and declining deviation between $A M I$ and $B M I$, as can be clearly seen in the diagram. The linear regression analysis of these data gives a clear correlation function as shown in equation 8:

$$
A M I=1.048 \cdot B M I+16.08
$$

The coefficient of determination of this linearization is still high, with $R^{2}=0.922$, although the number of male test subjects in this survey is rather small. Figure 6 shows the correlation of the Specific Body Surface (SBS) with the traditional BMI for male test subjects.

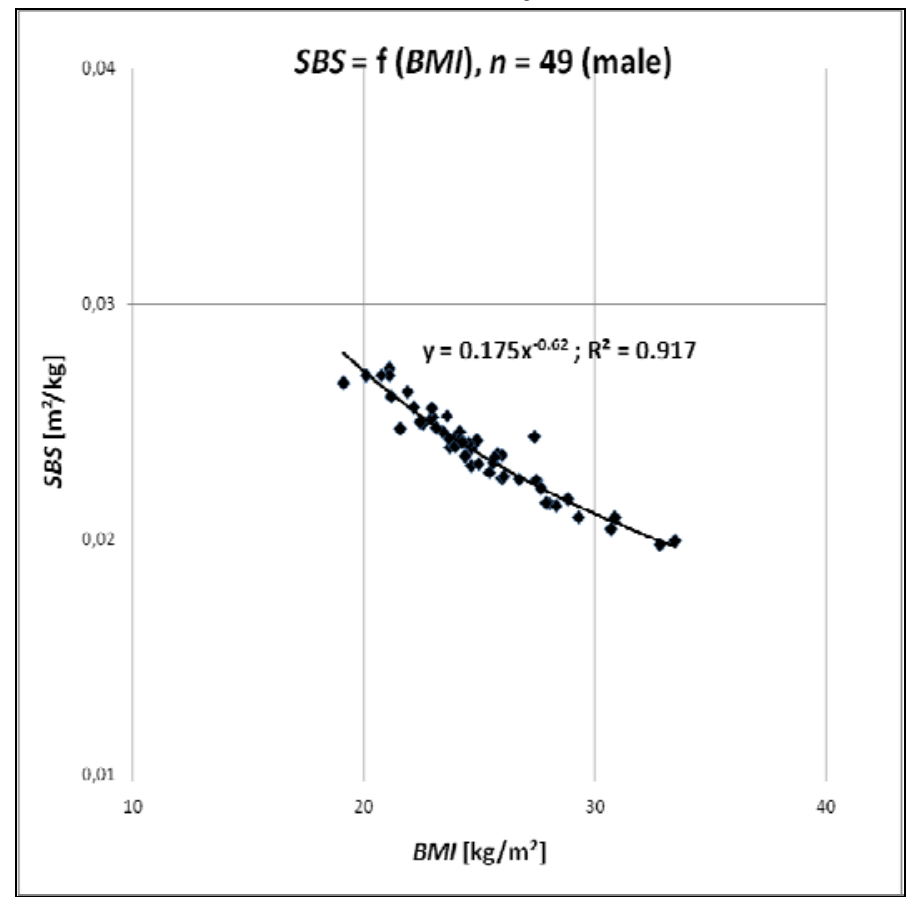

Fig. 6: Specific Body Surface (SBS) as a function of traditional BMI, $n=49$ (male)

Again, we find a declining hyperbolic relationship with regard to the dependency of the SBS on BMI. The associated nonlinear interpolation yields equation 9: 


$$
S B S=0.175 \cdot B M \Gamma^{0.62}
$$

The coefficient of determination of this nonlinear interpolation is quite high, with $R^{2}=0.917$, particularly with regard to the rather small number of male test subjects. The data of male test subjects show a maximum value of $S B S=0.027 \mathrm{~m}^{2} / \mathrm{kg}$ with low $B M I$ levels up to $0.02 \mathrm{~m}^{2} / \mathrm{kg}$ of adipose $B M I$. Again, the $S B S$ of trim figures turns out to be approximately $50 \%$ higher than stout figures. In general, however, the $S B S$ of men seem to be lower than the SBS of women. This result could be because of the significant difference between female and male physiques, which could lead to the higher values of SBS for women. However, because of the small number of male test subjects, this aspect of physical difference between men and women is plausible but not yet proven, and it should be verified by further 3D body scans.

To illustrate the difference between $A M I$ and the traditional $B M I$, it seems useful to calculate the ratio of $A M I$ to $B M I$ and to plot this ratio as function of $B M I$, separately for women and men (Fig. 7).

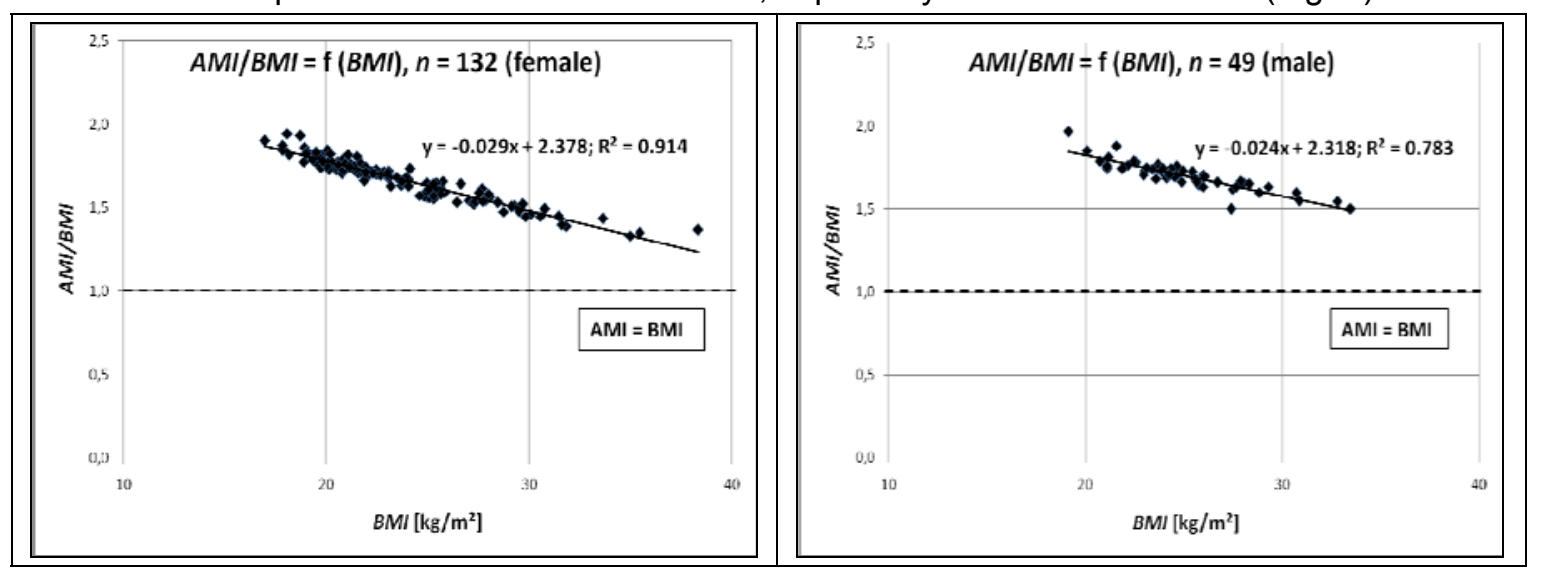

Fig. 7: Ratio of AMI to BMI as a function of BMI

Again, both charts demonstrate very clearly the deviation measured between $A M I$ and $B M I$ compared with the dotted line, $A M I I B M I=1$. Additionally, the deviation is not constant, but declining with an increasing BMI. Both observations have been expected before, and prove a discrepancy between the traditional $B M I$ and $A M I$, which declines with increasing $B M I$ as expected by the theory put forth. The appropriate linear functions and associated coefficients of determination are shown in equation 10 for female test subjects and equation 11 for male test subjects.

$$
\begin{array}{ll}
\text { Female: } & A M I / B M I=-0.029 \cdot B M I+2.378 \text { with } R^{2}=0.914 \\
\text { Male: } & A M I / B M I=-0.024 \cdot B M I+2.318 \text { with } R^{2}=0.783
\end{array}
$$

Both functions have a negative gradient, as was expected. As the $B M I$ increases, the ratio of $A M I / B M I$ advances closer to 1 . In other words, the $A M I$ approaches the $B M I$ in the case of very high $B M I$ values. Moreover, trim builds are clearly facing a severe deviation between $A M I$ and $B M I$.

In addition, the data points to lower values of female $A M I$ when compared with male $A M I$. This result corresponds with the observation of higher values of female SBS when compared with male SBS. As mentioned previously, this result could be due to the differences between the physiques of men and women, which could explain the higher values of SBS for women.

\section{Conclusions}

The measurement of the true body surface by means of the 3D-Body-Scan technology aids in the creation of the definition of the Area Mass Index (AMI) and the Specific Body Surface (SBS) for the test subjects. The comparison of the $A M I$ with the traditional $B M I$ highlights the expected systematic error of $B M I$. AMI, when compared with $B M I$, is not constant as it declines with increasing $B M I$. The hypothesis set forth, that the standard $B M I$ is a misleading measurement, can be quantified and varies between men and women.

Subjects with a trim build have values of SBS up to $50 \%$ higher when compared with subjects of stout build. The traditional calculation of $B M I$ does not consider this geometric effect. From the data presented, principal differences in the energy balance equation between trim and overweight figures are becoming clear. 
Stout subjects face two disadvantageous effects in terms of energy output. First, the ratio of body surface to body mass, SBS, is much smaller, which restricts the heat flux to the external environment. Hence, surplus energy has to be stored first as glycogen and later as fat deposit. Second, the fat deposit acts as an additional insulation for the body. These effects intensify each other so that overweight subjects tend to stay overweight because of the law of thermodynamics. Alternatively, trim figures tend to stay trim because their heat transferring surface is larger and because the heat insulation normally caused by a fat layer under the skin is very small.

The proposed use of the newly defined $A M I$ and $S B S$ instead of the historical $B M I$ considers these thermodynamic aspects. Thus, nutritional advice and recommendations for body mass management could be improved on the basis of these thermodynamic rules. The results of this research strongly support this idea. However, further investigation, with an increased number of test subjects, is needed to create a stronger data set and to verify the current results. Additionally, the results are valid for German people only, and conclusions cannot be made about other ethnicities. However, the results of this test series indicate that contrary to previous assumptions, significant differences exist between trim and stout builds as it relates to the principles of energy.

\section{References}

[1] Baehr HD, Kabelac S. Thermodynamik, Grundlagen und technische Anwendungen. 13. Aufl. Hamburg: Springer; 2006.

[2] Baehr HD, Stephan K. Wärme- und Stoffübertragung. 5. Aufl. Hamburg: Springer; 2006.

[3] World Health Organization. Physical Status: The Use and Interpretation of Anthropometry. World Health Organization (WHO), Geneva. 1995. Technical Report 854.

[4] Quetelet A: L'anthropometrie ou le mesure des differentes faculties de l'homme. Brussels; 1871.

[5] Breiner T: Dreidimensionale virtuelle Organismen [dissertation]. Fachbereich Informatik und Mathematik: Johann Wolfgang Goethe University Frankfurt; 2005.

[6] Hohensteiner Institute, Schloss Hohenstein, D-74357 Bönnigheim, http://www.hohenstein.de/.

[7] Human Solutions $\mathrm{GmbH}$, Europaallee 10, D-67657 Kaiserslautern, http://www.human-solutions.com/.

[8] SizeGERMANY [homepage on the internet]: SizeGERMANY - Die deutsche Reihenmessung. Hohensteiner Institute \& Human Solutions GmbH (2007). [Updated June 2007, cited July 2008] Available from: http://www.sizegermany.de 